\title{
Some heavy vector and tensor meson decay constants in light-front quark model
}

\author{
Chao-Qiang Geng ${ }^{1,2,3, a}$, Chong-Chung Lih ${ }^{3,4, b}$, Chuanhui Xia ${ }^{1, \mathrm{c}}$ \\ ${ }^{1}$ College of Materials Science and Engineering, Chongqing Jiaotong University, Chongqing 400074, China \\ 2 Department of Physics, National Tsing Hua University, Hsinchu 300, Taiwan \\ ${ }^{3}$ Physics Division, National Center for Theoretical Sciences, Hsinchu 300, Taiwan \\ ${ }^{4}$ Department of Optometry, Shu-Zen College of Medicine and Management, Kaohsiung Hsien 452, Taiwan
}

Received: 27 April 2016 / Accepted: 30 May 2016 / Published online: 6 June 2016

(C) The Author(s) 2016. This article is published with open access at Springerlink.com

\begin{abstract}
We study the decay constants $\left(f_{M}\right)$ of the heavy vector $\left(D^{*}, D_{s}^{*}, B^{*}, B_{s}^{*}, B_{c}^{*}\right)$ and tensor $\left(D_{2}^{*}, D_{s 2}^{*}, B_{2}^{*}, B_{s 2}^{*}\right)$ mesons in the light-front quark model. With the known pseudoscalar meson decay constants of $f_{D}, f_{D_{s}}, f_{B}, f_{B_{s}}$, and $f_{B_{C}}$ as the input parameters to determine the light-front meson wave functions, we obtain $f_{D^{*}, D_{s}^{*}, B^{*}, B_{s}^{*}, B_{c}^{*}}=\left(252.0_{-11.6}^{+13.8}\right.$, $\left.318.3_{-12.6}^{+15.3}, 201.9_{-41.4}^{+43.2}, 244.2 \pm 7.0,473.4 \pm 18.2\right)$ and $\left(264.9_{-9.5}^{+10.2}, 330.9_{-9.0}^{+9.9}, 220.2_{-46.2}^{+49.1}, 265.7 \pm 8.0,487.6 \pm\right.$ 19.2) $\mathrm{MeV}$ with Gaussian and power-law wave functions, respectively, while we have $f_{D_{2}^{*}, D_{s 2}^{*}, B_{2}^{*}, B_{s 2}^{*}}=\left(143.6_{-21.8}^{+24.9}\right.$, $\left.209.5_{-24.2}^{+29.1}, 80.9_{-27.7}^{+33.8}, 109.7_{-15.0}^{+15.7}\right) \mathrm{MeV}$ with only Gaussian wave functions.
\end{abstract}

\section{Introduction}

Meson decay constants contain useful information on the nonperturbative behavior of QCD between quarks and antiquarks inside mesons. In addition, the determinations of these helpful parameters can also be used to constrain the CKM mixing matrix elements in weak mesonic decays. In recent years, many heavy vector and tensor mesons have been experimentally discovered, such as the excited states of the charmed mesons [1-7], observed by Babar, Belle, CLEO, FOCUS, and LHCb Collaborations. Moreover, D0 [8,9] and CDF [10,11] Collaborations have confirmed the bottom states of $B_{1}(5721), B_{2}(5747), B_{s 1}(5830)$ and $B_{s 2}^{*}(5840)$. In some of these hadron states, the quantum numbers are $I\left(J^{P}\right)=\frac{1}{2}\left(2^{+}\right)$. The investigations of these particles are clearly important in hadron physics both theoretically and experimentally. The recent experimental results

\footnotetext{
a e-mail: geng@phys.nthu.edu.tw

be-mail: cclih@phys.nthu.edu.tw

c e-mail: chxia@cqjtu.edu.cn
}

on the parameters of these mesons would help us to understand the meson properties and the nonperturbative dynamics as well as the vacuum structure of QCD.

In the literature, the decay constants of heavy vector and tensor mesons are somewhat less discussed. In particular, compared to the scalar and pseudoscalar mesons, there are few theoretical works devoted to the analysis of the properties for the tensor mesons. The main purpose of this work is to examine the vector and tensor mesons decay constants simultaneously within the framework of the light-front quark model (LFQM), which has been widely used in the phenomenological study of meson physics. The LFQM is a good way for solving the nonperturbative problems of hadron physics and provides inside information as regards the internal structure of the bound state. The meson decay constant can be described by a two-point function and regarded as one of the simplest physical observable in the LFQM. This framework has been applied successfully to explain various properties of pseudoscalar and vector mesons [12-14].

The paper is organized as follows. In Sect. 2, we present the basic formalism of the LFQM. In Sect. 3, we show our numerical results on the decay constants in the LFQM. Our conclusions are given in Sect. 4.

\section{Formalism}

In the LFQM, a neutral meson wave function is constructed in terms of its constituent quark $q$ and anti-quark $\bar{Q}$ with the total momentum $p$ and spin $S$ as [15],

$$
\begin{aligned}
\left|M\left(p, S, S_{z}\right)\right\rangle= & \int\left[\mathrm{d} k_{1}\right]\left[\mathrm{d} k_{2}\right] 2(2 \pi)^{3} \delta^{3}\left(p-k_{1}-k_{2}\right) \\
& \times \sum_{\lambda_{1} \lambda_{2}} \Phi_{M}\left(k_{1}, k_{2}, \lambda_{1}, \lambda_{2}\right) b_{q}^{+}\left(k_{1}, \lambda_{1}\right) \mathrm{d}_{\bar{Q}}^{+} \\
& \times\left(k_{2}, \lambda_{2}\right)|0\rangle,
\end{aligned}
$$


where

$$
[\mathrm{d} k]=\frac{\mathrm{d} k^{+} \mathrm{d}^{2} k_{\perp}}{2(2 \pi)^{3}},
$$

$M$ represents for a $P$ (pseudoscalar) or $V$ (vector) or $T$ (tensor) meson, $\Phi_{M}$ is the wave function of the corresponding $q \bar{Q}$ and $k_{1(2)}\left(\lambda_{1(2)}\right)$ is the on-mass shell LF momentum (helicity) of the internal quark. In the momentum space, $\Phi_{M}$ can be expressed as a covariant form [12,13,16-18]

$$
\begin{aligned}
& \Phi_{M}\left(x, k_{\perp}\right)=\left(\frac{k_{1}^{+} k_{2}^{+}}{2\left[M_{0}^{2}-\left(m_{q}-m_{\bar{Q}}\right)^{2}\right]}\right)^{\frac{1}{2}} \\
& \times \bar{u}\left(k_{1}, \lambda_{1}\right) \Gamma v\left(k_{2}, \lambda_{2}\right) \phi_{M}\left(x, k_{\perp}\right), \\
& M_{0}^{2}=\frac{m_{q}^{2}+k_{\perp}^{2}}{x}+\frac{m_{\bar{Q}}^{2}+k_{\perp}^{2}}{1-x},
\end{aligned}
$$

where $\phi_{M}\left(x, k_{\perp}\right)$ describes the momentum distribution amplitude of the bound state for the $S$ - or $P$-wave meson, $\left(x, k_{\perp}\right)$ are LF relative momentum variables, defined by

$k_{1}^{+}=x p^{+}, \quad k_{2}^{+}=(1-x) p^{+}$,

$k_{1 \perp}=x p_{\perp}+k_{\perp}, \quad k_{2 \perp}=(1-x) p_{\perp}-k_{\perp}$,

and $\Gamma$ stands for

$\Gamma_{P}=\gamma_{5} \quad($ pseudoscalar, $S=0)$,

$\Gamma_{V}=i\left\{\not{\varepsilon}\left(S_{z}\right)-\frac{\hat{\varepsilon} \cdot\left(k_{1}-k_{2}\right)}{M_{0}+m_{q}+m_{\bar{Q}}}\right\} \quad$ (vector, $\left.S=1\right)$,

$\Gamma_{T}=i \frac{\hat{\varepsilon}^{\mu \nu}}{2}\left\{\gamma_{\mu}-\frac{\left(k_{1}-k_{2}\right)_{\mu}}{M_{0}+m_{q}+m_{\bar{Q}}}\right\}\left(k_{1}-k_{2}\right)_{\nu}$,

with

$\hat{\varepsilon}^{\mu}( \pm 1)=\left[\frac{2}{p^{+}} \vec{\varepsilon}_{\perp}( \pm 1) \cdot \vec{p}_{\perp}, 0, \vec{\varepsilon}_{\perp}( \pm 1)\right]$,

$\vec{\varepsilon}_{\perp}( \pm 1)=\mp(1, \pm i) / \sqrt{2}$,

$\hat{\varepsilon}^{\mu}(0)=\frac{1}{M_{0}}\left(\frac{-M_{0}^{2}+p_{\perp}^{2}}{p^{+}}, p^{+}, p_{\perp}\right)$.

There are several phenomenological light-front wave functions to describe the possible hadronic structures in the literature. In our work, we shall use the Gaussian-type and power-law wave functions, given by [19-23]

$$
\begin{aligned}
\phi_{P}\left(x, k_{\perp}\right) & =\phi_{V}\left(x, k_{\perp}\right)=N \sqrt{\frac{1}{N_{c}} \frac{\mathrm{d} k_{z}}{\mathrm{~d} x}} \exp \left(-\frac{\vec{k}^{2}}{2 \omega^{2}}\right), \\
& =N[x(1-x)]^{1 / n}\left[\frac{\omega^{2}}{\left(\mathcal{A}^{2}+k_{\perp}^{2}\right)+\omega^{2}}\right], \\
\phi_{T}\left(x, k_{\perp}\right) & =\sqrt{\frac{2}{\omega^{2}}} \phi_{P}\left(x, k_{\perp}\right),
\end{aligned}
$$

respectively, where $\omega$ is the scale parameter, $N_{c}$ is the number of colors, $N=4\left(\pi / \omega^{2}\right)^{\frac{3}{4}}, \vec{k}=\left(k_{\perp}, k_{z}\right), k_{z}$ is defined through

$x=\frac{E_{q}+k_{z}}{E_{q}+E_{\bar{Q}}}, \quad 1-x=\frac{E_{\bar{Q}}-k_{z}}{E_{q}+E_{\bar{Q}}}, \quad E_{i}=\sqrt{m_{i}^{2}+\vec{k}^{2}}$,

by

$k_{z}=\left(x-\frac{1}{2}\right) M_{0}+\frac{m_{q}^{2}-m_{\bar{Q}}^{2}}{2 M_{0}}, \quad M_{0}=E_{q}+E_{\bar{Q}}$,

$\mathrm{d} k_{z} / \mathrm{d} x=E_{q} E_{\bar{Q}} / x(1-x) M_{0}$, and $\mathcal{A}=m_{q} x+m_{\bar{Q}}(1-x)$.

The pseudoscalar and vector mesonic decay constants are defined by

$\left\langle 0\left|A^{\mu}\right| P\right\rangle=i f_{P} P^{\mu}$,

$\left\langle 0\left|V^{\mu}\right| V\right\rangle=f_{V} M_{V} \epsilon^{\mu}$,

where $A^{\mu}=\bar{q} \gamma^{\mu} \gamma_{5} Q$ and $V^{\mu}=\bar{q} \gamma^{\mu} Q$, respectively. For an ${ }^{3} P_{2}$ tensor meson with $J^{P C}=2^{++}$, the decay constant cannot be produced through the local $V-A$ and tensor currents. But it can be created from the local currents involving covariant derivatives [24-27]:

$\left\langle 0\left|J_{\mu \nu}\right| T\right\rangle=f_{T} M_{T}^{2} \epsilon_{\mu \nu}^{*}$,

where

$J_{\mu \nu}=\frac{i}{2}\left[\bar{q}_{1} \gamma_{\mu} \overleftrightarrow{D}_{\nu} q_{2}+\bar{q}_{1} \gamma_{\nu} \overleftrightarrow{D}_{\mu} q_{2}\right]$

and

$$
\begin{aligned}
& \overleftrightarrow{D}_{\mu}=\left[\vec{D}_{\mu}-\overleftarrow{D}_{\mu}\right] \\
& \vec{D}_{\mu}=\vec{\partial}_{\mu}-i \frac{g}{2} \lambda^{a} A_{\mu}^{a} \\
& \overleftarrow{D}_{\mu}=\overleftarrow{\partial}_{\mu}+i \frac{g}{2} \lambda^{a} A_{\mu}^{a}
\end{aligned}
$$

The polarization tensor $\epsilon_{\mu \nu}$ for a massive spin-2 particle can be constructed out of the polarization vector of a massive vector state $[27,28]$, given by

$$
\begin{aligned}
\epsilon_{\mu \nu}( \pm 2)= & \epsilon_{\mu}( \pm 1) \epsilon_{\nu}( \pm 1) \\
\epsilon_{\mu \nu}( \pm 1)= & \sqrt{\frac{1}{2}}\left[\epsilon_{\mu}( \pm 1) \epsilon_{\nu}(0)+\epsilon_{\mu}(0) \epsilon_{\nu}( \pm 1)\right], \\
\epsilon_{\mu \nu}(0)= & \sqrt{\frac{1}{6}}\left[\epsilon_{\mu}(+1) \epsilon_{\nu}(-1)+\epsilon_{\mu}(-1) \epsilon_{\nu}(+1)\right] \\
& +\sqrt{\frac{2}{3}} \epsilon_{\mu}(0) \epsilon_{\nu}(0) .
\end{aligned}
$$


From the definitions of the meson decay constants, one has

$$
\begin{aligned}
& \left\langle 0\left|A^{\mu}\right| P(p)\right\rangle=-\sqrt{N_{c}} \int \frac{\mathrm{d}^{4} k_{1}}{(2 \pi)^{4}} \Lambda_{P} \operatorname{Tr} \\
& \times\left[\Gamma_{P} \frac{i\left(-k / 1+m_{q}\right)}{k_{1}^{2}-m_{q}^{2}+i \epsilon} A^{\mu} \frac{i\left(\not p-k / 1+m_{\bar{Q}}\right)}{\left(p-k_{1}\right)^{2}-m_{\bar{Q}}^{2}+i \epsilon}\right], \\
& \left\langle 0\left|V^{\mu}\right| V(p)\right\rangle=-\sqrt{N_{c}} \int \frac{\mathrm{d}^{4} k_{1}}{(2 \pi)^{4}} \Lambda_{V} \operatorname{Tr} \\
& \times\left[\Gamma_{V} \frac{i\left(-k / 1+m_{q}\right)}{k_{1}^{2}-m_{q}^{2}+i \epsilon} V^{\mu} \frac{i\left(\not p-k / 1+m_{\bar{Q}}\right)}{\left(p-k_{1}\right)^{2}-m_{\bar{Q}}^{2}+i \epsilon}\right], \\
& \left\langle 0\left|J_{\mu \nu}\right| T(p)\right\rangle=-\sqrt{N_{c}} \int \frac{\mathrm{d}^{4} k_{1}}{(2 \pi)^{4}} \Lambda_{T} \operatorname{Tr} \\
& \times\left[\Gamma_{T} \frac{i\left(-k_{1}+m_{q}\right)}{k_{1}^{2}-m_{q}^{2}+i \epsilon} J^{\mu \nu} \frac{i(\not p-k / 1}{\left(p-m_{\bar{Q}}\right)}\right],
\end{aligned}
$$

where $\Lambda_{M}$ is a vertex function, related to the momentum distribution amplitude of the $q \bar{Q}$ Fock state. From Eqs. (3) and (15), we find the vertex function as

$\Lambda_{M}=\left(\frac{k_{1}^{+} k_{2}^{+}}{2\left[M_{0}^{2}-\left(m_{q}-m_{\bar{Q}}\right)^{2}\right]}\right)^{\frac{1}{2}} \phi_{M}\left(x, k_{\perp}\right)$,

where we have used the light-front variables in Eq. (4). Then the explicit expressions of the meson decay constants are given by [29-32]

$$
\begin{aligned}
f_{P}= & 4 \frac{\sqrt{3 N_{c}}}{\sqrt{2}} \int \frac{\mathrm{d} x \mathrm{~d}^{2} k_{\perp}}{2(2 \pi)^{3}} \phi_{P}\left(x, k_{\perp}\right) \frac{\mathcal{A}}{\sqrt{\mathcal{A}^{2}+k_{\perp}^{2}}}, \\
f_{V}= & 4 \frac{\sqrt{3 N_{c}}}{\sqrt{2}} \int \frac{\mathrm{d} x \mathrm{~d}^{2} k_{\perp}}{2(2 \pi)^{3}} \phi_{V}\left(x, k_{\perp}\right) \frac{1}{\sqrt{\mathcal{A}^{2}+k_{\perp}^{2}}} \\
& \times\left\{x(1-x) M_{0}^{2}+m_{q} m_{Q}+k_{\perp}^{2}\right. \\
& \left.+\frac{\mathcal{B}}{2 W}\left[\frac{m_{q}^{2}+k_{\perp}^{2}}{1-x}-\frac{m_{Q}^{2}+k_{\perp}^{2}}{x}-(1-2 x) M_{0}^{2}\right]\right\}, \\
f_{T}= & 4 \frac{\sqrt{N_{c}}}{\sqrt{2}} \int \frac{\mathrm{d} x \mathrm{~d}^{2} k_{\perp}}{2(2 \pi)^{3}} \phi_{T}\left(x, k_{\perp}\right) \frac{1}{x(1-x) \sqrt{\mathcal{A}^{2}+k_{\perp}^{2}}} \\
& \times\left\{2 k_{\perp}^{2}\left[k_{\perp}^{2}(2 x-1)^{2}+A^{2}\right]+(2 x-1)\left(k_{\perp}^{2}+m_{q} m_{Q}\right)\right. \\
& \times\left[(x-1) m_{q}^{2}+x m_{Q}^{2}+(2 x-1) k_{\perp}^{2}\right] \\
& +\frac{1}{2 W}\left[16 k_{\perp}^{4} x(1-x)\left(m_{q}+m_{Q}\right)+(1-2 x)^{2}\right.
\end{aligned}
$$

$$
\begin{aligned}
& \times\left(x\left(m_{q}+m_{Q}\right)\left(k_{\perp}^{2}+m_{q} m_{Q}\right)-m_{Q}\left(k_{\perp}^{2}+m_{q}^{2}\right)\right) \\
& \left.\left.\times\left(k_{\perp}^{2}(2 x-1)+m_{q}^{2}(x-1)+m_{Q}^{2} x\right)\right]\right\},
\end{aligned}
$$

where $\mathcal{A}=m_{q} x+m_{\bar{Q}}(1-x), \mathcal{B}=m_{q} x-m_{Q}(1-x)$, and $W=M_{0}+m_{q}+m_{\bar{Q}}$.

\section{Numerical results}

\subsection{Vector meson decay constants}

In the numerical calculation, we take the known decay constants of the pseudoscalar mesons $(P)$ and quark masses to evaluate the scalar parameters of $\omega_{P}$. For the meson wave functions, we first use the Gaussian-type wave function in Eq. (7a) and then the power-law one in Eq. (7b). For the latter, we only briefly summarize our results. We start from the decay constants of $f_{D}$ and $f_{D_{s}}$ from the PDG [33], given by

$f_{D}=204 \pm 5 \mathrm{MeV}, \quad f_{D_{s}}=257.5 \pm 4.6 \mathrm{MeV}$.

By using the first equation in Eq. (17) with the Gaussiantype wave function in Eq. (7a), taking the decay constants in Eq. (18) and inputting the quark masses of $m_{u}=m_{d}=0.25$ and $m_{s}=0.38$ in $\mathrm{GeV}$, we obtain the parameters $\omega_{D}$ and $\omega_{D_{s}}$ as functions of the charm quark mass $m_{c}$, shown in Fig. 1. In Fig. 2, by assuming the parameters of $\omega_{D^{*}}$ and $\omega_{D_{s}^{*}}$ are same as $\omega_{D}$ and $\omega_{D_{s}}$ with $m_{u, s}=(0.25,0.38) \mathrm{GeV}$, we plot the decay constants of $f_{D^{*}}$ and $f_{D_{s}^{*}}$ as functions of $m_{c}$ in the LFQM, respectively. From the figure, we see that the decay constants decrease with $m_{c}$ but the changes are mild. Consequently, from Fig. 2 with $m_{c}=1.5-1.8 \mathrm{GeV}$, we find $f_{D^{*}}=252.0_{-11.6}^{+13.8} \mathrm{MeV}, \quad f_{D_{s}^{*}}=318.3_{-12.6}^{+15.3} \mathrm{MeV}$,

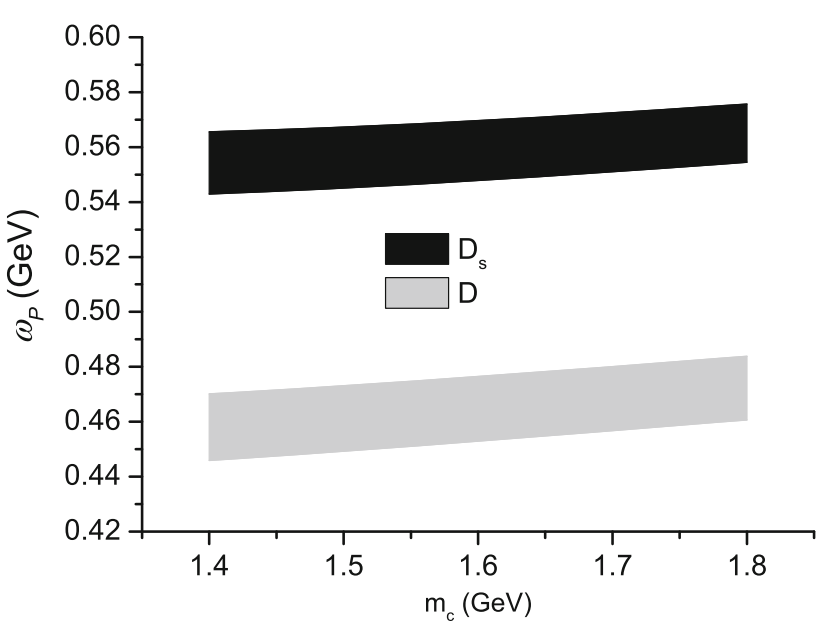

Fig. 1 Scalar parameters $\omega_{P}\left(P=D\right.$ and $\left.D_{s}\right)$ as functions of $m_{c}$ in the LFQM with $m_{q}=0.25$ and $m_{s}=0.38$ in $\mathrm{GeV}$ 


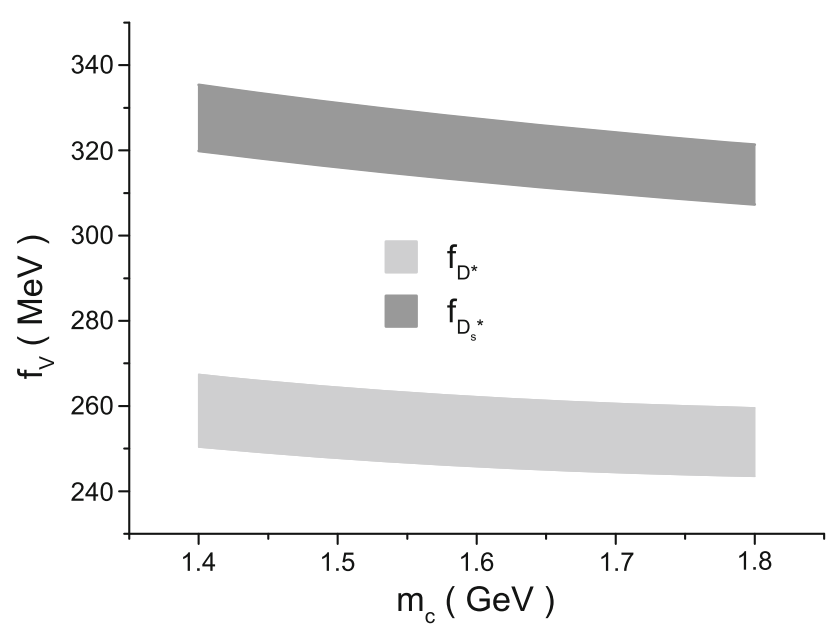

Fig. $2 f_{D^{*}}$ and $f_{D_{s}^{*}}$ as functions of $m_{c}$ in the LFQM

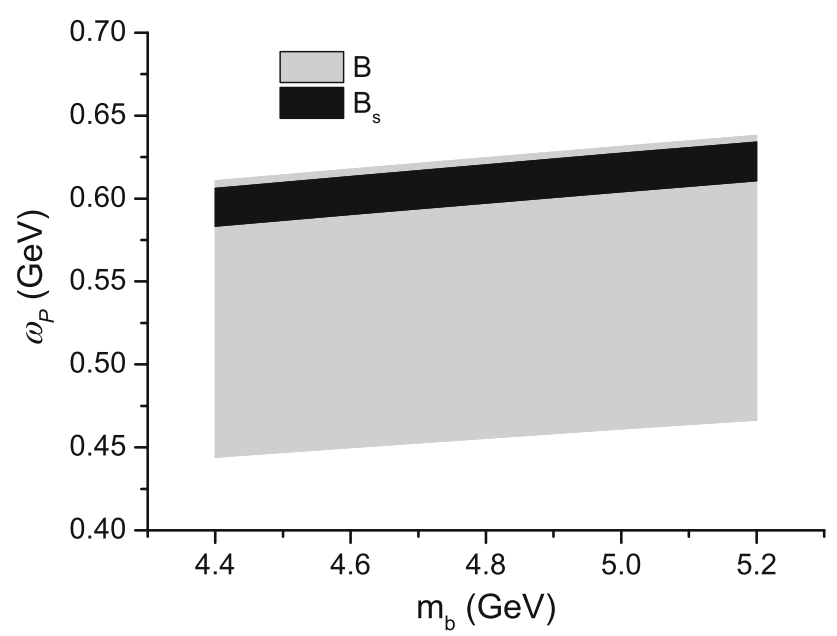

Fig. $3 \omega_{P}\left(P=B\right.$ and $\left.B_{s}\right)$ as functions of $m_{b}$ in the LFQM with $m_{q}=0.25$ and $m_{s}=0.38$ in GeV and the decay constants in Eq. (21)

which lead to the ratios of the vector and pseudoscalar meson decay constants as

$\frac{f_{D^{*}}}{f_{D}}=1.232_{-0.064}^{+0.074}, \quad \frac{f_{D_{s}^{*}}}{f_{D_{s}}}=1.236_{-0.054}^{+0.063}$,

respectively. Note that the uncertainties in Eqs. (19) come from those of Eq. (18) and $m_{c}$, while the errors in Eq. (20) result from the combinations of those in Eqs. (18) and (19).

From the Belle experimental results $[34,35]$ and the lattice QCD calculations [36] of $f_{B}, f_{B_{s}}$, and $f_{B_{c}}$ [39], given by

$f_{B}=185 \pm 35 \mathrm{MeV}, \quad f_{B_{s}}=224 \pm 5 \mathrm{MeV}$,

$f_{B_{c}}=434 \pm 15 \mathrm{MeV}$,

we can fix $\omega_{B}, \omega_{B_{s}}$, and $\omega_{B_{c}}$, respectively. Our results are shown in Figs. 3 and 4 with $m_{u, s, c}=(0.25,0.38,1.5) \mathrm{GeV}$. In Figs. 5 and 6, we present the decay constants of $f_{B^{*}}, f_{B_{s}^{*}}$, and $f_{B_{c}^{*}}$ as functions of $m_{b}$ in the LFQM.

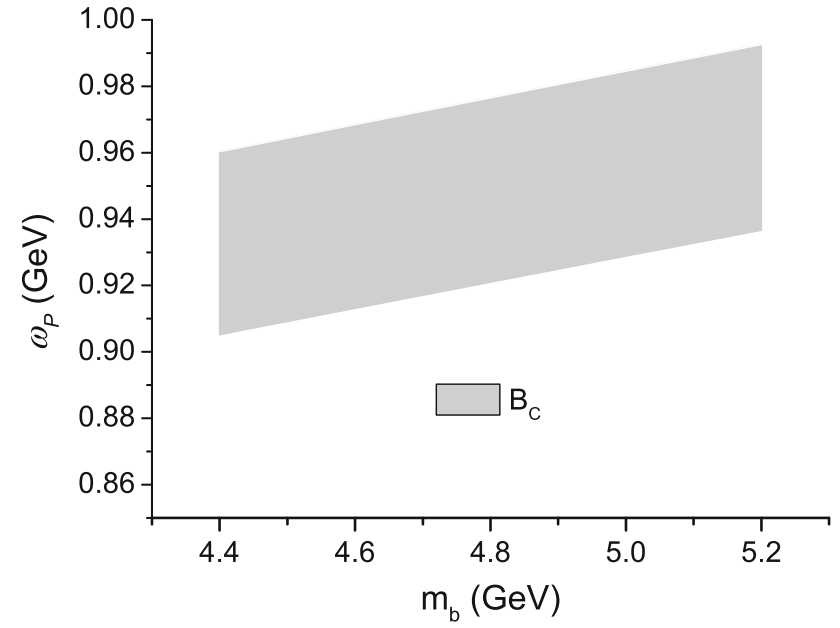

Fig. 4 Scalar parameters $\omega_{P}\left(B_{c}\right)$ as functions of $m_{b}$ in the LFQM with $m_{c}=1.5$ in $\mathrm{GeV}$

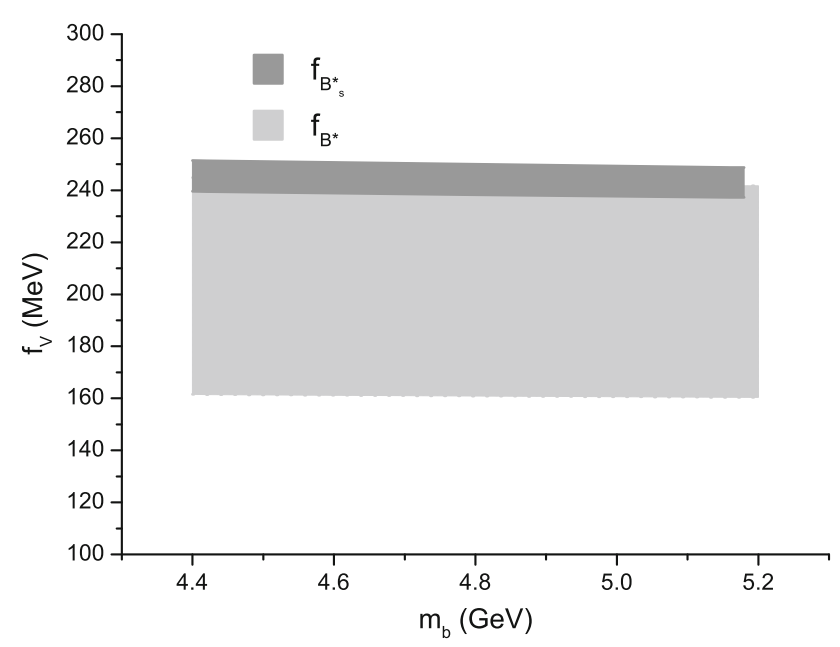

Fig. $5 f_{B^{*}}$ and $f_{B_{s}^{*}}$ as functions of $m_{b}$ in the LFQM.

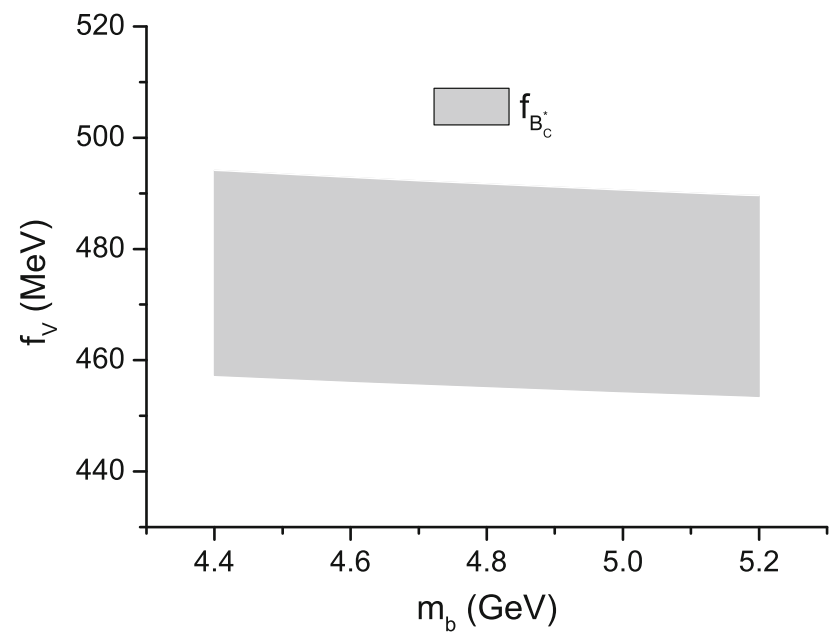

Fig. $6 f_{B_{c}^{*}}$ as functions of $m_{b}$ in the LFQM 
Table 1 Vector meson decay constants $f_{V}\left(V=D^{*}, D_{s}^{*}, B^{*}, B_{s, c}^{*}\right)$ in $\mathrm{MeV}$ in this work with (i) Gaussian and (ii) power-law meson wave functions and other theoretical calculations in Refs. [37-46]

\begin{tabular}{llllllll}
\hline & (i) & (ii) & LFQM [37] & Lattice QCD & QCDSR & QCDSR & QCDSR [44-46] \\
\hline$f_{D^{*}}$ & $252.0_{-11.6}^{+13.8}$ & $264.9_{-9.5}^{+10.2}$ & $259.6 \pm 14.6$ & $278 \pm 16[38]$ & $263 \pm 21[40]$ & $252.2 \pm 22.7[42]$ & $242_{-12}^{+20}$ \\
$f_{D_{s}^{*}}$ & $318.3_{-12.6}^{+15.3}$ & $330.9_{-9.0}^{+9.9}$ & $338.7 \pm 29.7$ & $311 \pm 9[38]$ & $308 \pm 21[40]$ & $305.5 \pm 27.3[42]$ & $293_{-14}^{+19}$ \\
$f_{B^{*}}$ & $201.9_{-41.4}^{+43.2}$ & $220.2_{-46.2}^{+49.1}$ & $225 \pm 38$ & $175 \pm 6[39]$ & $213 \pm 18[40]$ & $181.8 \pm 13.7[43]$ & $210_{-12}^{+10}$ \\
$f_{B_{s}^{*}}$ & $244.2 \pm 7.0$ & $265.7 \pm 8.0$ & $313 \pm 67$ & $213 \pm 7[39]$ & $255 \pm 19[40]$ & $225.6 \pm 18.5[43]$ & $251_{-16}^{+14}$ \\
$f_{B_{c}^{*}}$ & $473.4 \pm 18.2$ & $487.6 \pm 19.2$ & 387 & $422 \pm 13[39]$ & $384 \pm 32[41]$ & - & - \\
\hline
\end{tabular}

Obviously, these decay constants are insensitive to the change of $m_{b}$ as seen from the figures.

Similarly, we can derive the ranges of the decay constants $f_{B^{*}}$ and $f_{B_{s}^{*}}$ to be

$f_{B^{*}}=201.9_{-41.4}^{+43.2} \mathrm{MeV}, \quad f_{B_{s}^{*}}=244.2 \pm 7.0 \mathrm{MeV}$,

$f_{B_{c}^{*}}=473.4 \pm 18.2 \mathrm{MeV}$.

Note that the large error in Eq. (22) for $f_{B^{*}}$ originates from the one in Eq. (21) for $f_{B}$.

Subsequently, we get the ratios of the vector and pseudoscalar meson decay constants as

$\frac{f_{B^{*}}}{f_{B}}=1.09_{-0.30}^{+0.31}, \quad \frac{f_{B_{s}^{*}}}{f_{B_{S}}}=1.09 \pm 0.04$,

$\frac{f_{B_{c}^{*}}}{f_{B_{C}}}=1.09 \pm 0.06$.

In Table 1, we summarize our results with both Gaussian and power-law meson wave functions for the vector meson decay constants. In the table, we also show the other related theoretical values in the literature [37-46]. From the table, we find that our numerical values with the power-law wave functions are slightly higher than those with the Gaussian ones. In addition, we can see that our results for $f_{D_{(s)}^{(*)}, B_{(s)}^{(*)}}$ are consistent with those from the Lattice QCD [38] and QCD sum rules (QCDSR) in Refs. [40,42] but larger than the ones in Ref. [43].

We note that $f_{B_{(s)}^{*}} / f_{B}<1$ in Ref. [43]. For $f_{B_{c}^{*}}$, our predicted values are all larger than those in Refs. [39,41]. By comparing with Ref. [37], we see that our predictions for $f_{D^{*}}, f_{D_{s}^{*}}$, and $f_{B^{*}}$ are consistent each other within errors, but those for $f_{B_{s}^{*}}$ and $f_{B_{c}^{*}}$ are not. The main reasons for the differences are that the author in Ref. [37] used a different set of input parameters such as quark masses and decay constants of the pseudoscalar mesons.

Finally, we remark that if we take the sharp parameters $\omega_{V}$ of the vector mesons to be different from $\omega_{P}$ of the pseudoscalar ones, e.g. $\omega_{V} \sim(1+5 \%) \omega_{P}$, the corresponding vector meson decay constants will increase about $5 \%$ for the same set of input parameters. It is clear that our assumption of $\omega_{V} \sim \omega_{P}$ is a consequence of the heavy quark limit, in which $f_{P}=f_{V}$ is expected [47-49], so that it may only be applied to the heavy mesons as it obvious breaks down for the light mesons, such as the case of $\pi$ and $\rho$.

\subsection{Tensor meson decay constants}

Similar to the vector meson cases, if we take the parameters of $\omega_{T}$ to be the same as the corresponding ones for the pseudoscalar mesons, we may calculate the decay constants of the tensor mesons $D_{2}^{*}, D_{s 2}^{*}, B_{2}^{*}$, and $B_{s 2}^{*}$. In this part of the study, we shall concentrate on the Gaussian-type of the meson wave functions in Eq. (7a). Note that the relation in Eq. (7c) has been demonstrated only with the Gaussian wave functions [50]. Explicitly, we obtain

$$
\begin{aligned}
& f_{D_{2}^{*}}=143.6_{-21.8}^{+24.9} \mathrm{MeV}, \quad f_{D_{s 2}^{*}}=209.5_{-24.2}^{+29.1} \mathrm{MeV}, \\
& f_{B_{2}^{*}}=80.9_{-27.7}^{+33.8} \mathrm{MeV}, \quad f_{B_{s 2}^{*}}=109.7_{-15.0}^{+15.7} \mathrm{MeV},
\end{aligned}
$$

where $m_{u, s, c, b}=0.25,0.38,1.6$, and 4.8 in $\mathrm{GeV}$ have been used to evaluate the center values. Consequently, we find the ratios of the two related tensor meson decay constants to be

$\frac{f_{D_{s 2}^{*}}}{f_{D_{2}^{*}}}=1.5 \pm 0.3, \quad \frac{f_{B_{s 2}^{*}}}{f_{B_{2}^{*}}}=1.4_{-0.5}^{+0.6}$.

In Figs. 7 and 8, we show the tensor decay constants of $D_{2, s_{2}}$ $\left(B_{2, s_{2}}\right)$ as functions of $m_{c(b)}$.

One can see that the decay constants are enhanced if $m_{c(b)}$ increases.

In Table 2, we list our results for the tensor meson decay constants in the LFQM along with those in QCDSR [51]. From the table, we observe that our predicted value for $D_{s 2}^{*}$ is close to that in QCDSR, whereas the other ones are about $20 \%$ smaller. It is interesting to note that our results in the LFQM match with those in QCDSR if larger quark masses of $m_{c, b}$ are used.

\section{Conclusions}

We have studied the decay constants of the heavy vector $\left(D^{*}, D_{s}^{*} B^{*}, B_{s}^{*}, B_{c}^{*}\right)$ and tensor $\left(D_{2}^{*}, D_{s 2}^{*}, B_{2}^{*}, B_{s 2}^{*}\right)$ mesons in the LFQM. In our study, we have used the known 


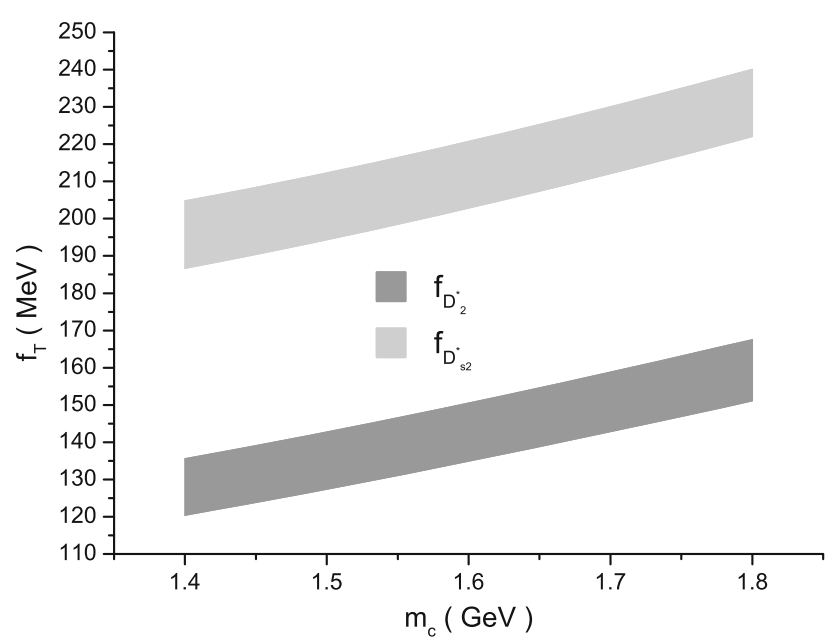

Fig. $7 f_{D_{2}^{*}}$ and $f_{D_{s_{2}}^{*}}$ as functions of $m_{c}$ in the LFQM

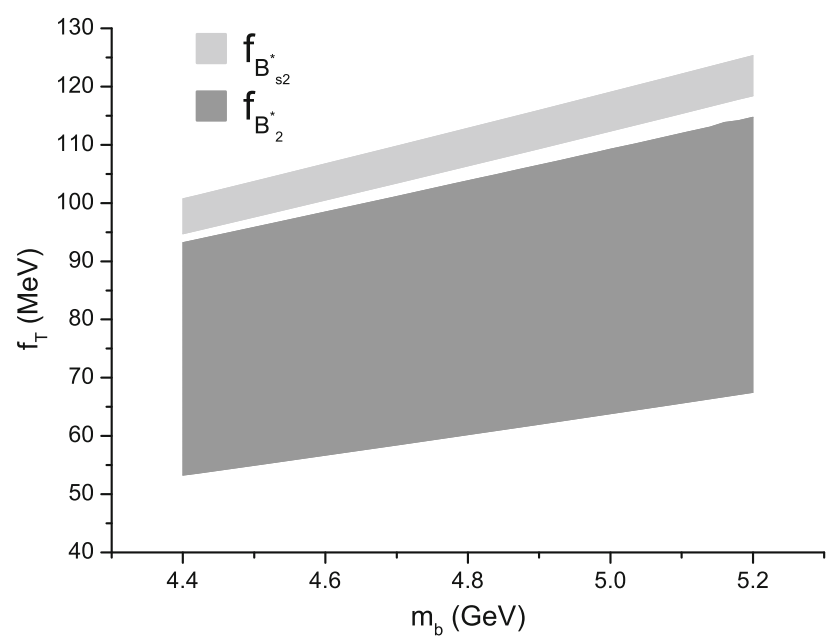

Fig. $8 f_{B_{2}^{*}}$ and $f_{B_{s_{2}}^{*}}$ as functions of $m_{b}$ in the LFQM

Table 2 Tensor meson decay constants of $f_{D_{2}^{*}}, f_{D_{s 2}^{*}}, f_{B_{2}^{*}}$, and $f_{B_{s 2}^{*}}$ $(\mathrm{MeV})$ in the LFQM and QCDSR [51]

\begin{tabular}{lll}
\hline & LFQM & QCDSR [51] \\
\hline$f_{D_{2}^{*}}$ & $143.6_{-21.8}^{+24.9}$ & $183 \pm 20$ \\
$f_{D_{s 2}^{*}}$ & $209.5_{-24.2}^{+29.1}$ & $222 \pm 22$ \\
$f_{B_{2}^{*}}$ & $80.9_{-27.7}^{+33.8}$ & $111 \pm 10$ \\
$f_{B_{s 2}^{*}}$ & $109.7_{-15.0}^{+15.7}$ & $134 \pm 11$ \\
\hline
\end{tabular}

pseudoscalar meson decay constants of $f_{D}, f_{D_{s}}, f_{B}, f_{B_{s}}$, and $f_{B_{c}}$ and quark mass $m_{u, d, s}$ and $m_{c(b)}$ as the input parameters to determine the values of the scale parameters of $\omega_{P}$ in the light-front wave functions. By taking $\omega_{D_{s}^{*}}$ and $\omega_{B_{s, c}^{*}}$ in both Gaussian and power-law wave functions being the same as the corresponding $\omega_{D_{s}}$ and $\omega_{B_{s, c}}$, we have calculated the decay constants of the vector $D_{(s)}^{*}$ and $B_{(s, c)}^{*}$ mesons, respectively. Explicitly, we have found that $f_{D^{*}, D_{s}^{*}, B^{*}, B_{s}^{*}, B_{c}^{*}}=\left(252.0_{-11.6}^{+13.8}, 318.3_{-12.6}^{+15.3}, 201.9_{-41.4}^{+43.2}\right.$, $244.2 \pm 7.0,473.4 \pm 18.2)$ and $\left(264.9_{-9.5}^{+10.2}, 330.9_{-9.0}^{+9.9}\right.$, $\left.220.2_{-46.2}^{+49.1}, 265.7 \pm 8.0,487.6 \pm 19.2\right) \mathrm{MeV}$ with Gaussian and power-law wave functions, respectively. Similarly, we have obtained $f_{D_{2}^{*}, D_{s 2}^{*}, B_{2}^{*}, B_{s 2}^{*}}=\left(143.6_{-21.8}^{+24.9}, 209.5_{-24.2}^{+29.1}\right.$, $\left.80.9_{-27.7}^{+33.8}, 109.7_{-15.0}^{+15.7}\right) \mathrm{MeV}$ with only Gaussian wave functions.

Acknowledgments The work was supported in part by National Center for Theoretical Sciences, National Science Council (NSC-101-2112M-007-006-MY3 and NSC-102-2112-M-471-001-MY3) and MoST (MoST-104-2112-M-007-003-MY3).

Open Access This article is distributed under the terms of the Creative Commons Attribution 4.0 International License (http://creativecomm ons.org/licenses/by/4.0/), which permits unrestricted use, distribution, and reproduction in any medium, provided you give appropriate credit to the original author(s) and the source, provide a link to the Creative Commons license, and indicate if changes were made.

Funded by $\mathrm{SCOAP}^{3}$.

\section{References}

1. E.S. Swanson, Phys. Rept. 429, 243 (2006)

2. B. Aubert et al. [BABAR Collaboration], Phys. Rev. Lett. 90, 242001 (2003)

3. P. Krokovny et al. [Belle Collaboration], Phys. Rev. Lett. 91, 262002 (2003)

4. D. Besson et al. [CLEO Collaboration], Phys. Rev. D 68, 032002 (2003)

5. B. Aubert et al. [BABAR Collaboration], Phys. Rev. D 69, 031101 (2004)

6. J. Link et al. [FOCUS Collaboration], Phys. Lett. B 586, 11 (2004)

7. R. Aaij et al. [LHCb Collaboration], Phys. Lett. B 698, 14 (2011)

8. V.M. Abazov et al., Phys. Rev. Lett. 99, 172001 (2007)

9. V. Abazov et al., Phys. Rev. Lett. 100, 082002 (2008)

10. T. Aaltonen et al., Phys. Rev. Lett. 102, 102003 (2009)

11. T. Aaltonen et al., Phys. Rev. Lett. 100, 082001 (2008)

12. W. Jaus, Phys. Rev. D 41, 3394 (1990)

13. W. Jaus, Phys. Rev. D 44, 2851 (1991)

14. W. Jaus, Phys. Rev. D 60, 054026 (1999)

15. C.C. Lih, C.Q. Geng, Phys. Rev. C 85, 018201 (2012)

16. K.G. Wilson, T.S. Walhout, A. Harindranath, W.M. Zhang, R.J. Perry, S.D. Glazek, Phys. Rev. D 49, 6720 (1994)

17. C.Q. Geng, C.C. Lih, W.M. Zhang, Phys. Rev. D 57, 5697 (1998)

18. C.Q. Geng, C.C. Lih, W.M. Zhang, Phys. Rev. D 62, 074017 (2000)

19. C.Q. Geng, C.C. Lih, W.M. Zhang, Mod. Phys. Lett. A 15, 2087 (2000)

20. C.C. Lih, C.Q. Geng, W.M. Zhang, Phys. Rev. D 59, 114002 (1999)

21. C.Q. Geng, C.C. Lih, C.C. Liu, Phys. Rev. D 62, 034019 (2000)

22. C.H. Chen, C.Q. Geng, C.C. Lih, C.C. Liu, Phys. Rev. D 75, 074010 (2007)

23. C.-W. Hwang, Phys. Lett. B 530, 93 (2002)

24. K. Azizi, H. Sundu, J.Y. Sungu, N. Yinelek, Phys. Rev. D 88, 036005 (2013)

25. K. Azizi, H. Sundu, J. Y. Sungu, N. Yinelek, Phys. Rev. D88, 099901(E) (2013)

26. K. Azizi, H. Sundu, A. Trkan, E.V. Veliev, J. Phys. G 41, 035003 (2014)

27. H.-Y. Cheng, K.-C. Yang, Phys. Rev. D 83, 034001 (2011)

28. E.R. Berger, A. Donnachie, H.G. Dosch, O. Nachtmann, Eur. Phys. J. C 14, 673 (2000) 
29. H.Y. Cheng, C.Y. Cheung, C.W. Hwang, Phys. Rev. D 55, 1559 (1997)

30. H.-M. Choi, Phys. Rev. D 75, 073016 (2007)

31. H.-M. Choi, C.-R. Ji, Phys. Rev. D 75, 034019 (2007)

32. H.-M. Choi, C.-R. Ji, Phys. Rev. D 89, 033011 (2014)

33. K.A. Olive et al. (Particle Data Group), Chin. Phys. C 38, 090001 (2014)

34. I. Adachi et al. (Belle Collab), Phys. Rev. Lett. 110, 131801 (2013)

35. M.J. Baker, J. Bordes, C.A. Dominguez, J. Penarrocha, K. Schilcher, JHEP 1407, 032 (2014)

36. R.J. Dowdall et al. [HPQCD Collaboration], Phys. Rev. Lett. 110(22), 222003 (2013)

37. C.-W. Hwang, Phys. Rev. D 81, 114024 (2010)

38. D. Becirevic, F. Sanfilippo, S. Simula, C. Tarantino, JHEP 1202, 042 (2012)

39. B. Colquhoun, C.T.H. Davies, J. Kettle, J. Koponen, A.T. Lytle, Phys. Rev. D 91, 114509 (2015)
40. Z.G. Wang, Eur. Phys. J. C 75, 427 (2015)

41. Z.G. Wang, Eur. Phys. J. A 49, 131 (2013)

42. W. Lucha, D. Melikhov, S. Simula, Phys. Lett. B 735, 12-18 (2014)

43. W. Lucha, D. Melikhov, S. Simula, Phys. Rev. D 91, 116009 (2015)

44. P. Gelhausen, A. Khodjamirian, A.A. Pivovarov, D. Rosenthal, Phys. Rev. D 88, 014015 (2013)

45. P. Gelhausen, A. Khodjamirian, A.A. Pivovarov, D. Rosenthal, Phys. Rev. D 89, 099901(E) (2014)

46. P. Gelhausen, A. Khodjamirian, A.A. Pivovarov, D. Rosenthal, Phys. Rev. D 91, 099901(E) (2015)

47. M. Neubert, Phys. Rev. D 46, 1076 (1992)

48. H.Y. Cheng, C.K. Chua, C.W. Hwang, Phys. Rev. D 69, 074025 (2004)

49. C.W. Hwang, Phys. Rev. D 86, 094031 (2012)

50. M.A. DeWitt, H.-M. Choi, C.-R. Ji, Phys. Rev. D 68, 054026 (2003)

51. Z.-G. Wang, Z.-Y. Di, Eur. Phys. J. A 50, 143 (2014) 